\title{
Expression patterns of dishevelled-2 in different colon tissue segments in Hirschsprung's disease
}

\author{
DONG CHEN ${ }^{1}$, JIE MI $^{1}$, DONG-HUI FU ${ }^{2}$, WEI-LIN WANG ${ }^{1}$ and HONG GAO ${ }^{1}$ \\ Departments of ${ }^{1}$ Pediatric Surgery and ${ }^{2}$ Urinary Surgery, Shengjing Hospital of \\ China Medical University, Liaoning 110004, P.R. China
}

Received January 8, 2014; Accepted October 24, 2014

DOI: $10.3892 / \mathrm{mmr} .2014 .2932$

\begin{abstract}
Hirschsprung's disease (HSCR) is a congenital disorder characterized by an absence of enteric ganglion cells in the terminal regions of the gut during development. To date, the cause of HSCR remains unclear, although the pathogenesis of this complex disease is hypothesized to be influenced by numerous genetic and environmental factors. Dishevelled-2 (DVL-2) is a subtype of the dishevelled protein, which is known to be involved in embryonic development. In the present study, the pathogenesis of HSCR was investigated by measuring the expression of the DVL-2 gene and protein using reverse transcription-quantitative polymerase chain reaction (RT-qPCR), western blotting and immunohistochemistry staining in the aganglionic and ganglionic segments of colonic tissues in patients with HSCR. The results showed that the level of DVL- 2 mRNA in the aganglionic segments was 0.28 fold that of the ganglionic segments. Similarly, the protein expression of DVL-2 was lower $(11.31 \pm 2.23)$ in the aganglionic segments than that of the ganglionic segments $(35.21 \pm 2.66)$, as assessed by western blot analysis. Furthermore, immunohistochemical staining demonstrated that DVL-2 expression was significantly higher in the mucosal and submucosal layers from ganglionic colon segments compared with that from the aganglionic segments. The data suggest that the expression of DVL-2 in colon tissue segments may be important in the pathogenesis of HSCR.
\end{abstract}

\section{Introduction}

Hirschsprung's disease (HSCR), one of the most common congenital disorders, is characterized by the absence of intestinal ganglion cells in the myenteric and submucosal plexuses (1). The disease often presents with intestinal

Correspondence to: Professor Hong Gao, Department of Pediatric Surgery, Shengjing Hospital of China Medical University, 36 Sanhao Street, Shenyang, Liaoning 110004, P.R. China

E-mail: gaohong515@vip.sina.com

Key words: Hirschsprung's disease, dishevelled-2, gene and protein, expression obstruction and massive distension of the bowel following birth (2). The incidence is $\sim 1$ in 5,000 live births, and this figure is slightly higher in Asian people ( 28 per 100,000 live births). Although the causes of HSCR remain unclear, it is known to be a complex disease, which is influenced by numerous genetic and environmental factors. To date, mutations in the ret proto-oncogene (3), endothelin receptor B (4), endothelin 3 (5), glial derived neurotrophic factor (6) and sex determining region Y-box 10 (7), which are all known to be involved in the formation of the enteric nervous system, have been identified in patients with HSCR. However, changes in these genes do not account for all cases of HSCR. At present, unidentified genes, or combinations of genes, underlie as many as $50-70 \%$ of cases.

The Wnt signaling pathway is involved in a number of biological process, including cell proliferation, tumorigenesis and embryonic development $(8,9)$. The present study investigated the association between HSCR disease and the Wnt signaling pathway. It has previously been demonstrated that the WNT8A gene is involved in susceptibility to HSCR and is important in the development of this disease (10). The dishevelled (DVL) protein, which has three homologous subtypes, DVL-1, DVL-2 and DVL-3, is a cytoplasmic protein that is important in embryonic development, cell differentiation and tumor formation (11-13). It is also known to be a key part of the Wnt signaling pathway (14). A previous study showed that in patients with HSCR, the expression of DVL-1 and DVL-3 in aganglionic colon segments was higher than that in ganglionic segments (15). However, the correlation between expression of the DVL-2 protein and HSCR has not been confirmed. Therefore in the current study, differential expression of DVL-2 was detected using reverse transcription-quantitative polymerase chain reaction (RT-qPCR), western blotting and immunohistochemistry staining.

\section{Materials and methods}

Patients and specimens. This study was approved by the Ethics Committee of China Medical University (Ethical no. 2013PS07K; Liaoning, China). Colonic tissues were obtained from 50 patients (41 males and 9 females) with pathologically confirmed HSCR pre- or post-operative at Shengjing Hospital of China Medical University. HSCR was 
diagnosed by a barium enema X-ray and suction rectal biopsy. All patients with HSCR exhibited aganglionosis restricted to the sigmoid colon. Their ages ranged from 0.5 to 4.5 years old, with an average of 1.5 years. Based on the results of the barium enema and the intestinal morphology observed during and after the operation, the surgical specimens were divided into aganglionic colon segments and ganglionic colon segments. These classifications were further confirmed by histological examination of a frozen section of each segment. Aganglionic and ganglionic colon segment tissues were collected and identified by histopathological examination of hematoxylin and eosin (H\&E)-stained slides. Each tissue specimen was divided into two; one part was frozen at $-80^{\circ} \mathrm{C}$ for molecular analysis and the other was fixed in $10 \%$ neutral-formalin and embedded with paraffin.

Reagents and instruments. A biotin streptavidin detection system for immunohistochemical staining, and a DAB-0031/1031 kit were obtained from Fuzhou Maixin Biotech Co., Ltd. (Fuzhou, China). The PrimeScript RT reagent kit was obtained from Takara Biotechnology Co. Ltd. (Dalian, China). The electrophoresis apparatus was obtained from Bio-Rad Laboratories (Hercules, CA, USA). The mixed SYBR green kit from Takara Biotechnology was made for RT-qPCR conducted using a LightCycler ${ }^{\circledR} 480$ instrument (Roche Diagnostics, Basel, Switzerland).

Design and synthesis of primers. Human $\beta$-actin was used as an internal control and the length of the amplified fragment was 317 base pairs (bp). The other primers were constructed using the cDNA $\chi$ sequence of DVL-2, for which the length was $129 \mathrm{bp}$. These two pairs of primers were synthesized and purified by the Shanghai Biological Engineering Company (Shanghai, China). The primer sequences were as follows: Forward: 5'-AGA GCT ACG AGC TGC CTG AC-3' and reverse: 5'-AGC ACT GTG TTG GCG TAC AG-3' for human $\beta$-actin; and forward: 5'-CCA CCT TTA CCT CCT TTG-3' and reverse: 5'-CAC TAC TGA CTC GGT TTC TG-3' for human DVL-2.

$R N A$ extraction, $R T-q P C R)$. Tissues $(\sim 100 \mathrm{mg})$ from the aganglionic and ganglionic intestines were used for total RNA extraction using the RNA extraction reagent, TRIzol ${ }^{\circledR}$ (Invitrogen Life Technologies, Carlsbad, CA, USA), according to the manufacturer's instructions. The harvested RNA was diluted to a concentration of $1 \mu \mathrm{g} / \mu \mathrm{l}$, aliquoted and stored at $-80^{\circ} \mathrm{C}$. For cDNA synthesis, two reagent kits were used (One Step PrimeScript ${ }^{\circledR}$ mRNA cDNA Synthesis kit and PrimeScript ${ }^{\circledR}$ RT reagent kit, Takara Biotechnology Co., Ltd.).

RT-qPCR was performed in triplicate for each specimen using SYBR ${ }^{\circledR}$ Green PCR Master Mix in a LightCycler ${ }^{\circledR}$ (Roche Molecular Biochemicals, Co., Mannheim, Germany). The reference gene, $\beta$-actin (Takara Biotechnology, Co., Ltd.; DR3783), was used as an endogenous control. The following conditions were used: 5 min pre-denaturation at $95^{\circ} \mathrm{C}, 40$ cycles of $5 \mathrm{sec}$ denaturation at $95^{\circ} \mathrm{C}$ and $30 \mathrm{sec}$ annealing at $52.5^{\circ} \mathrm{C}$. Following the termination of PCR, the production was analyzed automatically by the Lightcycler system. The amplification process was followed by a melting curve analysis and the cycle threshold $(\mathrm{Ct})$ value was recorded. For the genes investigated here, one cycle change in $\mathrm{Ct}$ corresponded to a $2.1 \pm 0.2$ (standard error of the mean) change in RNA dilution. The relative expression of the gene was calculated by the $2^{-\Delta \Delta \mathrm{Ct}}$ method (16).

$H \& E$ and immunohistochemical staining. Diagnosis of HSCR was based on H\&E staining of ganglion cells. Review of surgical pathological reports following resection of the colonic segment confirmed whether the initial biopsy diagnosis of HSCR had been accurate. Aganglionic and ganglionic segments were categorized according to the results of H\&E staining (Fig. 1). Segments were fixed in $10 \%$ neutral-formalin and embedded in paraffin.

Immunohistochemistry (IHC) was performed on 5- $\mu \mathrm{m}$ sections obtained from formalin-fixed, paraffin embedded blocks, using a biotin streptavidin complex method. For antigen retrieval, slides were incubated in a microwave oven for $10 \mathrm{~min}$ in $0.01 \mathrm{~mol} / 1$ citrate buffer $(\mathrm{pH}=6$; Zhongshan Golden Bridge Biotechnology Co., Ltd., Beijing, China), followed by cooling at room temperature. Following incubation in 10\% normal goat serum (Beijing TransGen Biotech Co. Ltd., Beijing, China) in phosphate-buffered saline (PBS; Zhongshan Golden Bridge Biotechnology Co., Ltd.) for $30 \mathrm{~min}$ in order to block nonspecific binding sites, samples were incubated at $4^{\circ} \mathrm{C}$ overnight with a polyclonal rabbit anti-DVL-2 antibody (1:2,000; Millipore, Billerica, MA, USA; catalog no. AB5972). Samples were washed with PBS and then incubated with anti-rabbit IgG peroxidase-conjugated antibody (1:2,000; Beijing TransGen Biotech Co. Ltd.; catalog no. I10318) for $20 \mathrm{~min}$ at $37^{\circ} \mathrm{C}$ and washed by PBS. The final reaction product was stained with diaminobenzidine (Beijing TransGen Biotech Co. Ltd.). After 10 min washing with PBS, nuclei were counterstained with hematoxylin. The negative control was produced using an equivalent quantity of PBS in place of the rabbit anti-DVL-2. Brown and yellow deposition represented a positive reaction. Density and distribution were observed under a light microscope (Nikon E800, Nikon Corporation, Kanagawa, Japan). The density of the positively stained area was calculated, at x400 magnification, as the sum of the areas occupied by the positively stained area of the tissues. Images were captured and the area of staining in each image was calculated using a NISE Elements Basic Research (version 2.30, Kawasaki, Kanagawa, Japan) analysis system. Negative controls were performed using an equivalent quantity of PBS instead of rabbit anti-DVL-2. Two pathologists independently reviewed the IHC-stained slides and agreed on a diagnoses by consensus.

Western blot analysis. Colon tissue specimens ( $50 \mathrm{mg})$ were cut into small pieces using surgical scissors, sonicated in protein lysis buffer (Beijing TransGen Biotech Co. Ltd.) and centrifuged at $13,600 \times \mathrm{g}$ for $15 \mathrm{~min}$ at $4^{\circ} \mathrm{C}$. The protein concentration was measured using the Bradford method (17), specimens were adjusted to obtain the same protein concentration, and then aliquoted and stored at $-80^{\circ} \mathrm{C}$. Samples containing equal quantities of protein $(50 \mu \mathrm{g})$ were separated by sodium-dodecyl sulfate polyacrylamide gel electrophoresis on a $10 \%$ gel, and then electrotransferred onto polyvinylidene fluoride membranes (EMD Millipore). 
Table I. Relative quantity of DVL-2 mRNA in aganglionic and ganglionic segments of colon tissue.

\begin{tabular}{lccccc}
\hline Segment & DVL-2 average Ct value & $\beta$-actin average Ct value & $\Delta C t$ & $\Delta \Delta C t$ & Relative expression \\
\hline Ganglionic & $26.73 \pm 1.59$ & $23.94 \pm 1.95$ & 2.79 & 0.00 & 1.00 \\
Aganglionic & $28.57 \pm 1.03$ & $24.06 \pm 1.80$ & 4.51 & 1.82 & 0.28 \\
\hline
\end{tabular}

DVL-2, dishevelled-2; Ct, cycle threshold.
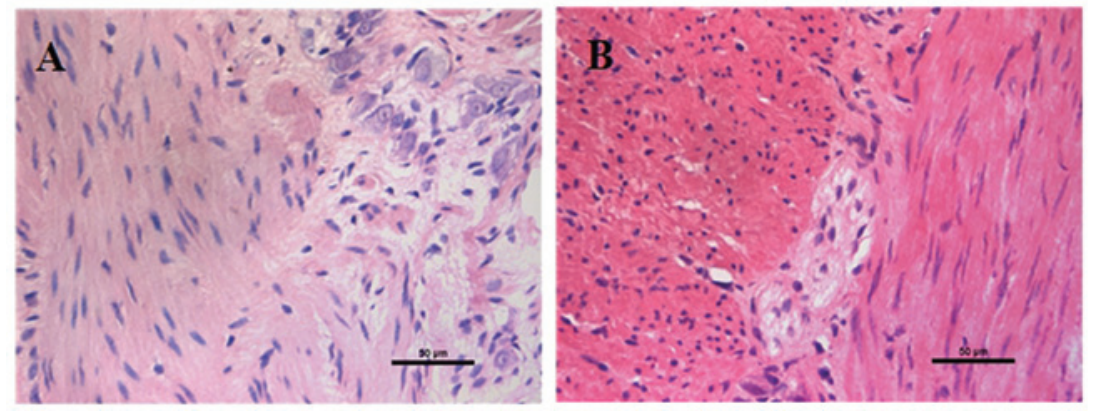

Figure 1. Photomicrographs of aganglionic and ganglionic intestine using hematoxylin and eosin staining. (A) Ganglionic colon segment tissue and (B) aganglionic colon segment tissue. (A) and (B): Magnification, x400; scale bar, $50 \mu \mathrm{m}$.

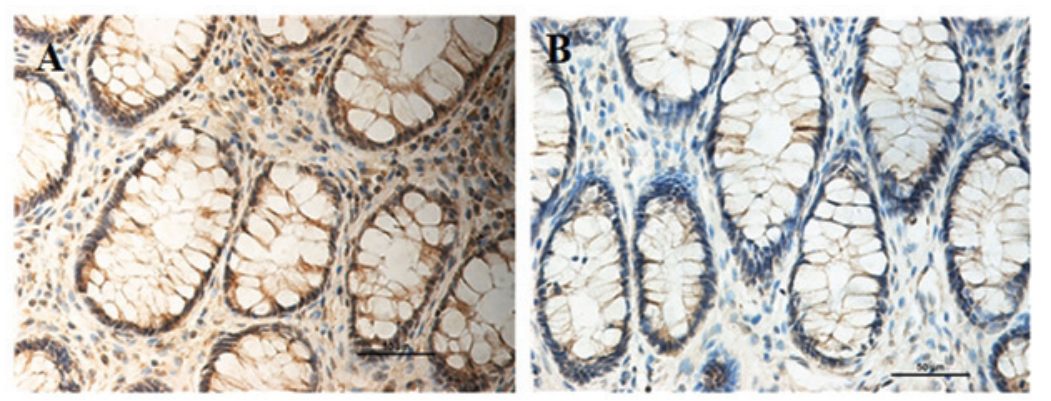

Figure 2. Expression of DVL-2 detected by immunohistochemistry in aganglionic and ganglionic colon segments. The brown-yellow depositions in the ganglionic segments were richer and more widespread in the submucosa, while those in the aganglionic segment were observed in a punctiform distribution. (A) Ganglionic colon segment tissue and (B) aganglionic colon segment tissue. (A) and (B): Magnification, x400; scale bar, $50 \mu \mathrm{m}$.

Blots were blocked with $5 \%$ non-fat for $1 \mathrm{~h}$ at $37^{\circ} \mathrm{C}$, followed by incubation with anti-DVL2 antibodies $(1: 1,000)$ overnight at $4^{\circ} \mathrm{C}$. The following day, the blots were washed in TBST, and incubated with goat anti-rabbit $\operatorname{IgG}$ (1:2,000 dilution; Beijing ZhongShan Jinqiao Biological Technology Co., Ltd., Beijing, China; catalog no. ZB-2301) for $90 \mathrm{~min}$ at $37^{\circ} \mathrm{C}$ prior to detection by enhanced chemiluminescence (Pierce Biotechnology, Inc., Rockford, IL, USA). The grayscale values of the DVL-2 band were normalized to the values of the corresponding $\beta$-actin band to determine the relative expression levels of the DVL-2 protein. Experiments were repeated three times, independently.

Statistical analysis. The Statistical Program for Social Sciences, version 13.0 (SPSS, Inc., Chicago, IL, USA), was used for statistical analysis. A t-test was used to compare the expression level of DVL-2 in aganglionic colon segments with that of the normal segments. Data are expressed as the mean \pm standard deviation. $\mathrm{P}<0.05$ was considered to indicate a statistically significant difference.

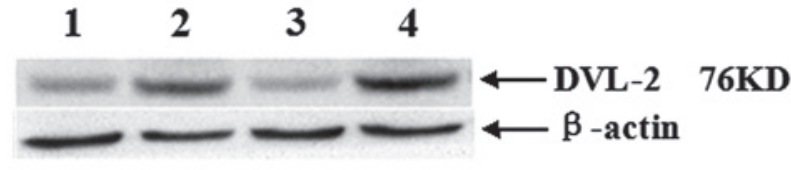

Figure 3. Representative western blot showing expression of the DVL-2 protein. The DVL-2 protein was detected as an $\sim 76 \mathrm{kDa}$ band on western blots of protein extracted from ganglionic and aganglionic colon segment tissues. The immunoblot shows a strong signal for the DVL-2 protein in the ganglionic tissues and a weak signal in the aganglionic tissue. Lines 1 and 3, DVL-2 proteins from the aganglionic segment; and lines 2 and 4, DVL-2 proteins from the ganglionic segment. DVL-2, dishevelled-2.

\section{Results}

$R T-q P C R$ analysis. The optical density value of RNA calculated by A260/A280 ranged from 1.8 to 2.0. In the analysis of RT-qPCR data, the mRNA expression levels of DVL-2 were normalized to the mRNA expression levels of $\beta$-actin in each specimen. The results showed that the mRNA expression 
levels of DVL-2 in the aganglionic segments were $72 \%$ lower than those in the ganglionic segments $(\mathrm{P}<0.03$; Table I).

IHC staining results. The aganglionic and ganglionic segments were first defined by $\mathrm{H} \& \mathrm{E}$ staining, which demonstrated the absence of focal ganglion cells (Fig. 1). Immunohistochemical staining was performed and positive reaction was predominantly detected in the mucosal and submucosal layers of the ganglionic colonic segments. The brown-yellow depositions observed in the ganglionic segments tissues were richer and more widespread in the submucosa. The distribution was reticulodromous in the circular muscular layer, while that of the aganglionic segment tissues was punctiform. DVL-2 immunoreactivity was greater in the ganglionic segments than the aganglionic segments (Fig. 2).

Western blot analysis. Expression of the DVL-2 protein was evaluated by western blotting with specific antibodies in samples from the same group of patients with HSCR. In accordance with the results from the RT-qPCR experiments, a significant decrease in the DVL-2 protein was detected in aganglionic segments compared with the matched ganglionic segments (Fig. 3). The protein level of DVL-2 was $11.31 \pm 2.23$ in the aganglionic colon segment, compared with $35.21 \pm 2.66$ in the ganglionic segments $\mathrm{P}<0.05$ ).

\section{Discussion}

HSCR is a congenital disorder, characterized by an absence of enteric neurons in the myenteric and submucosal plexuses of the terminal gastrointestinal tract. It usually presents with intestinal obstruction and massive distension of the proximal bowel (megacolon) following birth (2). To date, numerous genes have been reported to be involved in the etiology of HSCR, however, the mechanisms underlying the motility dysfunction observed in HSCR remain unclear. Furthermore, for patients with HSCR, a significant problem is that of persistent postoperative disturbances in bowel motility, even following surgery. The reasons for this phenomenon also remain to be elucidated. In the present study, RT-qPCR, IHC staining and western blotting were used to investigate the differential expression in mRNA and protein levels of DVL-2 between aganglionic and normal ganglionic colonic tissues, in order to gain information on bowel motility disturbance. Aganglionic and normal colonic segment tissues, derived from 50 patients with sporadic HSCR were analyzed. The results showed that the expression of DVL-2 mRNA in the aganglionic segments was lower than that in the ganglionic segments (Table I) and that these differences were statistically significant $(\mathrm{P}<0.05)$. Results of IHC experiments to assess the DVL-2 protein levels confirmed a significant reduction in DVL-2 expression in aganglionic colonic segments compared with the ganglionic segments.

As a member of the Wnt signaling pathway, DVL exerts its biological function predominantly through the canonical and non-canonical Wnt signaling pathways $(18,19)$. Within the central nervous system, DVL is widely expressed in early embryonic and postnatal nervous tissues, and is crucial for the formation of connections between neurons (11). When DVL-2 expression is inhibited, neuronal precursor cell proliferation is reduced. A previous study showed that in patients with HSCR, the expression of DVL-1 and DVL-3 in aganglionic colonic segments was greater than that in ganglionic segments (15). The different expressions between DVL-1, DVL-3 and DVL-2 in aganglionic colon segments may indicate that different subtypes of DVL gene have different roles in the development of the enteric nervous system. When the Wnt signaling pathway becomes abnormal, resulting in a reduced quantity of DVL-2, synapse formation is reduced. In order to restore this function, expression of DVL-1 and DVL-3 increases, stimulating synapse formation by increasing synaptic assembly, in order to promote normal development of ganglions.

There are a number of mechanisms regarding the role of DVL-2 in HSCR that require further investigation, including the mechanism by which a reduced expression of DVL-2 results in the development of HSCR. The present study demonstrated a differential expression of DVL-2 mRNA and protein between the aganglionic and the ganglionic colon segments, suggesting that DVL-2 may be an important factor in the pathogenesis of HSCR. The difference in expression of DVL-2 mRNA and protein between the aganglionic segments and the ganglionic segments provides a starting point for further investigation into the molecular basis of HSCR.

\section{Acknowledgements}

This study was supported by the Shenyang Science and Technology Plan Project (grant no. F13-316-1-01).

\section{References}

1. Amiel J, Sproat-Emison E, Garcia-Barcelo M, et al: Hirschsprung disease, associated syndromes and genetics: a review. J Med Genet 45: 1-14, 2008.

2. Whitehouse FR and Kernohan JW: Myenteric plexus in congenital megacolon; study of 11 cases. Arch Int Med (Chic) 82: 75-111, 1948.

3. Lantieri F, Griseri P and Ceccherini I: Molecular mechanisms of RET-induced Hirschsprung pathogenesis. Ann Med 38: 11-19, 2006.

4. Kusafuka T, Wang $\mathrm{Y}$ and Puri P: Novel mutations of the endothelin-B receptor gene in isolated patients with Hirschsprung's disease. Hum Mol Genet 5: 347-349, 1996.

5. Bidaud C, Salomon R, Van Camp G, et al: Endothelin-3 gene mutations in isolated and syndromic Hirschsprung disease. Eur J Hum Genet 5: 247-251, 1997.

6. Salomon R, Attié T, Pelet A, et al: Germline mutations of the RET ligand GDNF are not sufficient to cause Hirschsprung disease. Nat Genet 14: 345-347, 1996.

7. Pan ZW, Lou J, Luo C, Yu L and Li JC: Association analysis of the SOX10 polymorphism with Hirschsprung disease in the Han Chinese population. J Pediatr Surg 46: 1930-1934, 2011.

8. Martin BL and Kimelman D: Canonical Wnt signaling dynamically controls multiple stem cell fate decisions during vertebrate body formation. Dev Cell 22: 223-232, 2012.

9. Gurney A, Axelrod F, Bond CJ, et al: Wnt pathway inhibition via the targeting of Frizzled receptors results in decreased growth and tumorigenicity of human tumors. Proc Natl Acad Sci USA 109: 11717-11722, 2012.

10. Gao H, Chen D, Liu X, et al: Polymorphisms and expression of the WNT8A gene in Hirschsprung's disease. Int J Mol Med 32: 647-652, 2013.

11. Almuedo-Castillo M, Saló E and Adell T: Dishevelled is essential for neural connectivity and planar cell polarity in planarians. Proc Natl Sci Acad USA 108: 2813-2818, 2011.

12. Zhang X, Zhu J, Yang GY, et al: Dishevelled promotes axon differentiation by regulating atypical protein kinase C. Nat Cell Biol 9: 743-754, 2007. 
13. Pulvirenti T, Van Der Heijden M, Droms LA, et al: Dishevelled 2 signaling promotes self-renewal and tumorigenicity in human gliomas. Cancer Res 71: 7280-7290, 2011.

14. Gao $\mathrm{C}$ and Chen YG: Dishevelled: The hub of Wnt signaling. Cell Signal 22: 717-727, 2010.

15. Chen D, Mi J, Wu M, Wang W and Gao H: The expression of dishevelled gene in Hirschsprung's disease. Int J Clin Exp Pathol 6: 1791-1798, 2013.

16. Livak KJ and Schmittgen TD: Analysis of relative gene expression data using real-time quantitative PCR and the $2^{-\Delta \Delta c}$ method. Methods 25: 402-408, 2001.
17. Bradford MM: A rapid and sensitive method for the quantitation of microgram quantities of protein utilizing the principle of protein-dye binding. Anal Biochem 72: 248-54, 1976.

18. Liu C, Li Y, Semenov M, et al: Control of beta-catenin phosphorylation/degradation by a dual-kinase mechanism. Cell 108: 837-847, 2002

19. Habas R, Dawid IB and He X: Coactivation of Rac and Rho by Wnt/Frizzled signaling is required for vertebrate gastrulation. Genes Dev 17: 295-309, 2003. 